\title{
Microwave Photonics: Recent Programs at DARPA
}

\author{
Richard W. Ridgway ${ }^{1}$, Joshua A. Conway ${ }^{2}$ and Carl L. Dohrman ${ }^{3}$ \\ ${ }^{1}$ Strategic Technology Office, DARPA, Arlington VA 22203 \\ ${ }^{2}$ Microsystems Technology Office, DARPA, Arlington VA 22203 \\ ${ }^{3}$ Booz Allen Hamilton, Arlington VA 22203
}

\begin{abstract}
Microwave photonic components and systems are of significant interest to radar and communications systems used on military platforms. This paper highlights recent experimental results and system architectures aimed at moving microwave photonic component developments to system demonstrations and transitions to deployed military systems.
\end{abstract}

Keywords-Microwave photonics, noise figure, optical fiber links, spurious free dynamic range, electrooptic modulator.

\section{INTRODUCTION}

Multifunctional receivers, capable of performing communications, radar and electronic warfare functions, are of considerable interest to the military. The next generation of multifunctional receiver technology will require substantial gains in several key performance parameters, including increased frequency of operation, increased instantaneous bandwidth, increased dynamic range and reduced minimum detectable signal. Furthermore, there is a continued desire to reduce the system size, weight, power and cost. The application of microwave photonic components and links in such receivers has high potential to produce significant improvements to these performance parameters. Microwave photonic components operate at very high frequencies with very wide bandwidths [1], [2], [3]. Furthermore, microwave photonic technologies are compatible with channelization to simultaneously allow wide instantaneous bandwidths and exceptionally low minimum detectable signals. Finally, microwave photonics use optical fiber to transport the signals around the platform which reduces the RF loss and significantly reduces the cable size and weight. This paper aims to outline system performance metrics for receivers and provide a roadmap to improving system performance.

The three performance metrics of interest for a receiver include the bandwidth, the minimum detectable signal and the dynamic range. In general, the bandwidth and the noise figure influence the minimum detectable signal as well as the spurious free dynamic range (SFDR), as shown in Figure 1. The dashed line at $110 \mathrm{~dB} \mathrm{~Hz}^{2 / 3}$ is representative of the SFDR of state of the art electronic receiver. The solid line denotes the minimum detectable signal as a function of bandwidth for a typical X-Band electronic receiver chain with a noise figure of $5 \mathrm{~dB}$. To achieve the desired dynamic range of greater than $60 \mathrm{~dB}$ and minimum detectable signal of $-90 \mathrm{dBm}$ requires a receiver bandwidth of about 10 to $100 \mathrm{MHz}$. Channelization approaches, implemented using multiple parallel channels, can be used to increase the instantaneous bandwidth. However,

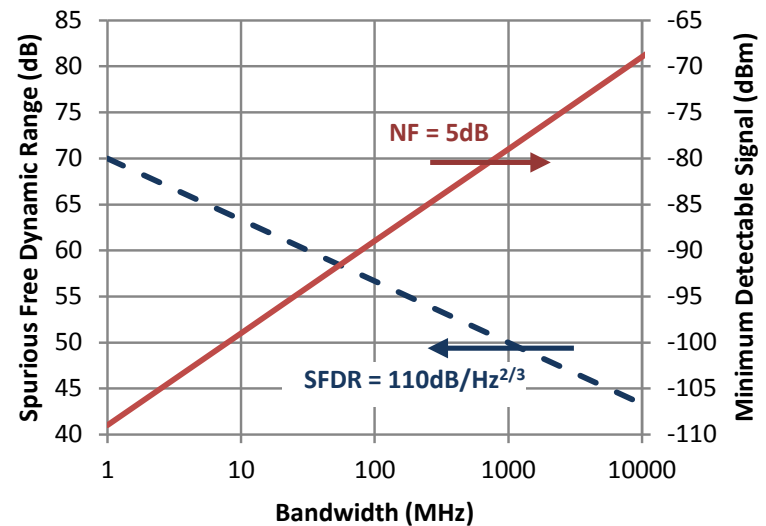

Fig. 1. Spurious free dynamic range and minimum detectable signal as a function of bandwidth for a typical RF receiver.

for electronic systems this often means replicating the entire receiver chain resulting in a bulky and heavy system, particularly as we move to higher frequency bands.

Microwave photonics has long held the promise of providing low noise, high bandwidth, light weight interconnections between radar and their remoted antennas [2]. While there are numerous components and architectures that have been used to create a microwave photonic link, the three dominant components that enable the effective use of microwave photonics include:

1. A low-noise, high power laser diode,

2. A low loss, low $\mathrm{V}_{\pi}$ electrooptic modulator, and

3. A high power, highly linear photodiode.

Over the last twenty years, DARPA has invested in numerous programs aimed specifically at the improvement and maturation of these three components. This paper will summarize some of the recent results and describe how these performance metrics could translate to system attributes of interest to military platforms.

\section{Microwave Photonic TeChNOLOGIES}

A block diagram of a microwave photonic link is shown in Figure 2. A low-noise, continuous-wave laser diode provides light to an electrooptic modulator, such as a lithium niobate modulator configured as a Mach-Zehnder interferometer. A microwave signal drives the electrooptic modulator which modulates the light that is directed over a single-mode fiber to a high power photodiode. This section summarizes the recent progress made on these three key microwave photonic components. 


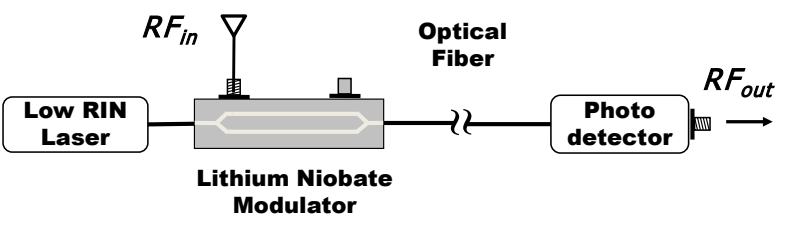

Fig. 2. Block diagram of a microwave photonics link.

\section{A. The Low-Noise Laser Diode}

Minimizing the relative intensity noise (RIN) of the laser diode is an essential step in achieving the desired link noise figure and spurious free dynamic range. Under the DARPA NEW-HIP program, a DFB laser was designed to achieve low RIN and high power for use in a high-performance microwave photonic link at a wavelength of $1550 \mathrm{~nm}$. The fabricated devices from this design were reported to achieve output power over $200 \mathrm{~mW}$, with side-mode suppression ratio above $60 \mathrm{~dB}$ and RIN less than $-164 \mathrm{~dB} / \mathrm{Hz}$ over a frequency range of 0.1 to $20 \mathrm{GHz}$ [4].

\section{B. The Electrooptic Modulator}

The optical transfer function of a Mach-Zehnder electrooptic modulator can be written as [5]:

$$
P_{\text {out }}(t)=\frac{T_{f f} P_{1}}{2}\left(1+\cos \left(\frac{\pi\left(V_{B}+v_{m} \sin \left(\omega_{m} t\right)\right)}{V_{\pi}}\right)\right)
$$

where $T_{f f}$ is the fiber-to-fiber throughput of the modulator, $P_{l}$ is the optical input power in $\mathrm{mW}, V_{B}$ is the dc bias voltage, $V_{\pi}$ is the voltage needed to induce a $\pi$ phase shift, $v_{m}$ is the input modulation voltage, and $\omega_{m}$ is the modulation frequency in radians per second. Although there has been a great deal of interest and exploration of the role of the bias condition of the modulator [6], the most common bias point is at quadrature, where the second-order distortion is minimized. When the modulator is biased at quadrature, the third-order distortion limits the SFDR, as will be discussed later.

The two critical metrics for the electrooptic modulator are $T_{f f}$ and $V_{\pi}$ DARPA has explored these two parameters in a number of programs, including ULTRA-T/R [6], P-STAR, and TROPHY. Figure 3 summarizes the measured $V_{\pi}$ as a function of frequency [6].

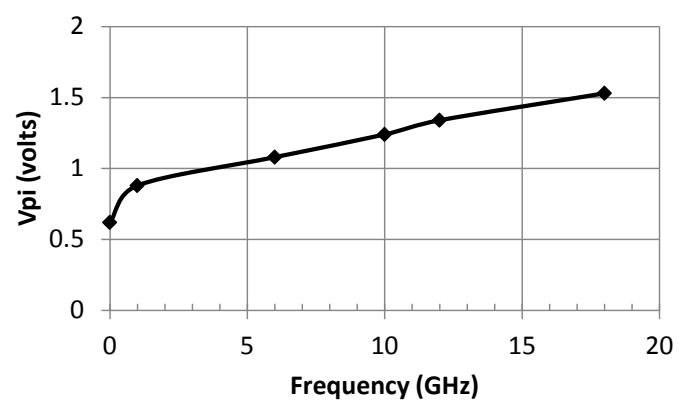

Fig. 3. Measured $V_{\pi}$ as a function of frequency. [6]

\section{Photodetectors}

When operating in the linear region of the photodiode response, the output current of the photodiode is given by:

$$
i_{\text {det }} \text { ? } r_{d} P_{\text {out }}
$$

where $r_{d}$ is the detector responsivity in $\mathrm{A} / \mathrm{W}$. Neglecting the frequency response of the photodiode and electrooptic modulator, the output RF signal is given by:

$$
P_{R F \text { ॠut }} \text { ? } i_{\text {det }}^{2} R_{L} \text { ? }
$$

where $R_{L}$ is the load resistor. To address the high-power, high-speed, high-linearity requirements of microwave photonic receivers, the unitraveling carrier (UTC) photodiode has established itself as the leading photodiode architecture for high-performance receivers. Under the DARPA TROPHY program, significant advances in high power, high linearity UTC photodiodes were demonstrated [7]. Specific developments include the demonstration of UTC photodiodes with $\mathrm{RF}$ output power up to $0.75 \mathrm{~W}$ at $15 \mathrm{GHz}$ with an output third-order intercept point (OIP3) of $>55 \mathrm{dBm}$. The maximum power level was achieved using InP/InGaAs-based UTC photodiode structures bonded to a high thermal conductivity substrate.

\section{Noise Figure}

The noise figure is a critically important metric of the link. This is particularly true if one does not plan to use a low noise amplifier between the antenna and the electrooptic modulator. The noise figure of a link is given by [5]:

$$
\left.N F \text { 回国国国 [0 回 } \frac{n_{\text {add }}}{g_{i} n_{i n}}\right]
$$

where $n_{\text {in }}$ is the input noise $\left(n_{\text {in }}=k T B\right), n_{\text {add }}$ is the noise added by the link and $g_{i}$ is the intrinsic gain of the analog link. For a Mach Zehnder interferometer, $g_{i}$ is given by:

$$
g_{i} \text { 目 }\left(\frac{T_{f f} P_{1} \pi R_{s}}{2 V_{\pi}}\right)^{2} r_{d}^{2}
$$

Recognizing that there are two resistors (the load resistor of the Mach Zehnder and the detector load resistor) that contribute to the system noise, the noise figure can be rewritten as [5]:

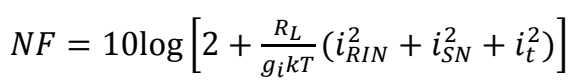

where $i_{R I N}^{2} \mathbb{Q}_{S N}^{2}$ and $i_{t}^{2}$ are the noise-like currents associated with laser RIN, shot noise, and thermal noise, respectively.

Laser RIN tends to be the dominant noise current for microwave photonic links operating with high average photodetector current [5]. Figure 4 shows the calculated noise figure as a function of laser RIN for a link with high optical power $\left(P_{l}=30 \mathrm{dBm}\right)$, and low optical loss through the electrooptic modulator $\left(T_{f f}=0.5\right)$. The three curves show $V_{\pi}=4 \mathrm{~V}, V_{\pi}=2 \mathrm{~V}$, and $V_{\pi}=1 \mathrm{~V}$. 


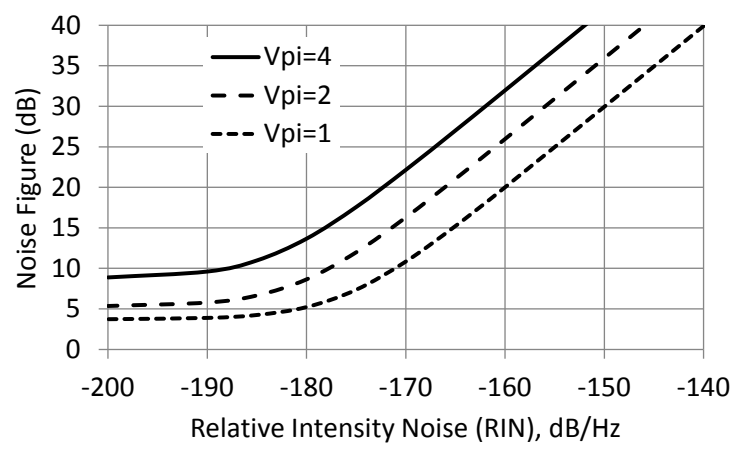

Fig. 4. Calculated link noise figure as a function of laser RIN.

\section{E. Spurious-Free Dynamic Range}

For amplitude-modulated links of the type depicted in Figure 2, the component that fundamentally limits the performance of the photonic link is the electrooptic modulator. When biased at quadrature, the cosine response shown in the modulator transfer function (Eq. (1)) will generate third-order nonlinearities as the input signals become large. Equations (1), (2) and (3) can be used to calculate the microwave photonic link's transfer function. Figure 5 shows a calculated transfer function for a link with component metrics shown in Table 1.

The SFDR of the microwave photonic link is limited by the third-order intermodulation distortion (IMD3) as is given by [8]:

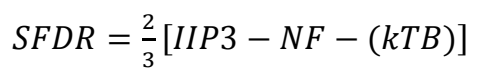

where IIP3 is the input third-order distortion intercept point. For an electrooptic modulator in a Mach Zehnder interferometer configuration, IIP3 increases with increasing $\mathrm{V}_{\pi}$. In Figure 5, IIP3 was calculated to be $13.7 \mathrm{dBm}$.

\section{F. Photonic Downconversion}

While photonic links can achieve large SFDR, the dynamic range of a receiver chain is typically limited by the mixer. To push system performance, the TROPHY program investigated wideband photonic downconversion [9]. As experiment demonstrated, mixing in the optical regime provided significantly reduced spurious signals over state-ofthe-art electronic mixers. This performance improvement is due to the optical filtering of harmonics before the mixing process. The technique also employed balanced detection that rejected common mode noise such as RIN and ASE beat noise. Using commercially available components, the TROPHY team was able to demonstrate photonic downconversion at X-Band with a SFDR near $120 \mathrm{~dB} \mathrm{~Hz}^{2 / 3}$ [9].

\section{G. Channelized Receivers}

The tradeoff between bandwidth and minimum detectable signal often leads to the use of channelized receivers where
Table 1. Parameters used for link model.

\begin{tabular}{|l|l|}
\hline Parameter & Value \\
\hline Laser Power, $P_{1}$ & $1 \mathrm{~W}$ \\
\hline Modulator throughput loss, $T_{f f}$ & $5 \mathrm{~dB}$ \\
\hline$V_{\pi}$ & 1.7 volts \\
\hline Detector responsivity, $r_{d}$ & $0.9 \mathrm{~A} / \mathrm{W}$ \\
\hline Modulator load resistance, $R_{m}$ & $50 \mathrm{ohms}$ \\
\hline Detector load resistance, $R_{l}$ & $50 \mathrm{ohms}$ \\
\hline
\end{tabular}

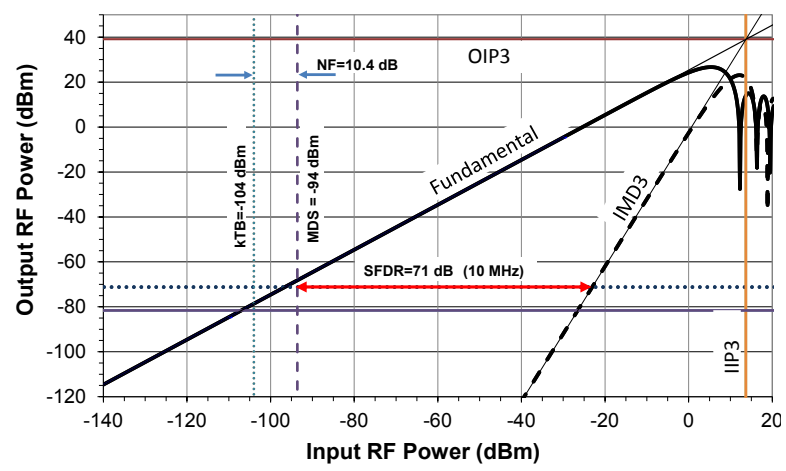

Fig. 5. Calculated RF Transfer curve for a microwave photonic link.

multiple channels, each operating over a narrow bandwidth, work in parallel to increase the instantaneous bandwidth. For microwave receivers, this usually means replicating the bandpass filter, the LNA, the cables, the mixer and the digitizer, leading to a bulky and heavy receiver system. For microwave photonic systems, channelized systems can share the broadband electrooptic modulator and optical fiber and have the channelization occur in the optical domain near the receiver, leading to a significant reduction in complexity and weight [10]. Efforts at DARPA to minimize the optical loss in planar lightwave circuits and to create tunable optical filters with bandwidths as low as $50 \mathrm{MHz}$ provide opportunities to build microwave photonic, channelized receivers with wide instantaneous bandwidths and large spurious free dynamic ranges.

\section{AREAS FOR FURTHER DEVELOPMENT}

Over the last few years, significant progress has been made at reducing the laser noise, reducing the electrooptic modulator's $\mathrm{V}_{\pi}$, and increasing the power handling capability and linearity of photodiodes. In addition, the frequency response of the modulators and detectors continues to increase. There has also been considerable progress in tunable optical filters and low-loss waveguide circuits.

However, there are significant challenges translating these component improvements into desirable system performance metrics. Table 2 summarizes the desired system performance for a microwave channelized receiver. Achieving this broad instantaneous bandwidth within the Ka band along with the 
Table 2. Desired System Performance Metrics for a MicrowavePhotonic-Based Channelized Receiver

\begin{tabular}{|l|l|}
\hline Parameter & Value \\
\hline Frequency Band & Ka Band \\
\hline Instantaneous Bandwidth & $>5 \mathrm{GHz}$ \\
\hline Dynamic Range & $>70 \mathrm{~dB}$ \\
\hline Receiver Sensitivity & $<-90 \mathrm{dBm}$ \\
\hline
\end{tabular}

desired dynamic range and receiver sensitivity is a significant challenge. One approach to achieving these challenging metrics is to use photonic links in a channelized array, as shown schematically in Figure 6. Here, each link achieves high dynamic range and improved sensitivity by limiting the bandwidth of each channel. Photonic downconversion is used to mix the RF modulated optical signal down to baseband where it is digitized with a low bandwidth analogto-digital converter.

There are a variety of planar lightwave circuit designs that might be considered to implement the channelized receiver. Optical interleavers, arrayed waveguide gratings, directional couplers and tunable optical filters are some of the components that might comprise such a circuit. Technical challenges include:

- The creation of narrowband optical filters needed to perform RF filtering while still in the optical domain,

- Ultra low-loss optical waveguides to keep the microwave photonic link losses at a minimum, and

- Minimizing the microwave phase distortion across the channelized bands.

Two DARPA programs that addressed some of these issues were iPhoD, which focused on reducing the loss in integrated optical waveguides, and PhASER that investigated tunable RF filters and signal processing functions carried out in the optical domain. The iPhoD program made significant progress in reducing the loss of on-chip optical waveguides, demonstrating losses of $0.08 \mathrm{~dB} / \mathrm{m}$ through a $27 \mathrm{~m}$ integrated waveguide, with losses of $0.037 \mathrm{~dB} / \mathrm{m}$ achieved in waveguide resonators [11].

Another area in need of further development for photonicbased antenna remoting is the antenna-to-electrooptic modulator interface. Issues such as impedance matching and alternative electrooptic antenna feeds that provide a more efficient interface between the antenna and electrooptic modulator could minimize the receiver noise figure and maximize the SFDR.

\section{CONCLUSIONS}

Over the last twenty years DARPA has invested significantly in microwave photonics. DARPA's Microsystems Technology Office has been a leader in developing microwave photonic components, architectures and links. Using these technical advances for microwave antenna remoting, photonic downconversion, and channelized

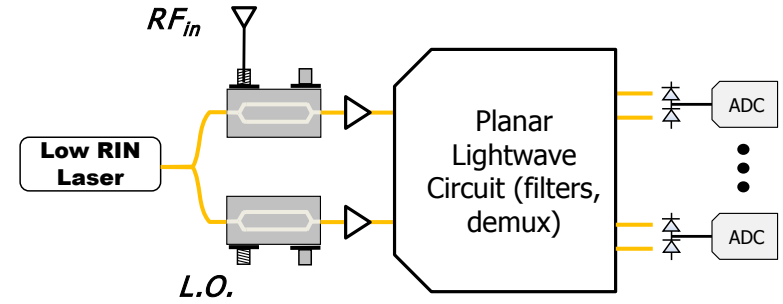

Fig. 6. Microwave-photonic-based channelized receiver.

receivers could significantly improve the performance metrics for the military's advanced receivers, including frequency range, instantaneous bandwidth, dynamic range and receiver sensitivity. The next step is to move microwave photonic technologies from these experimental efforts and demonstrations to deployed military systems.

\section{REFERENCES}

1. E. Ackerman, et al. "RF-over-fiber links with very low noise figure" J. Lightwave Tech. 26 (15), p. 2441, 2008.

2. S. Pappert, "RF Photonics: Status, Challenges and Opportunities", Avionics, Fiber-Optics and Photonics Technology Conference, Paper TuA4, 2011.

3. R. Esman, et al. "Photonics for microwave generation, transmission and processing", Optical Fiber Communications Conf., Paper OTuE5, 2009

4. Y.-G. Zhao, et al., "High-power and low-noise DFB semiconductor lasers for RF photonic links," Avionics, Fiber-Optics and Photonics Technology Conference (AVFOP), 2012.

5. C. Cox, Analog Optical Links. Cambridge, U.K.: Cambridge Univ. Press, 2004.

6. E. Ackerman, et al. "Signal-to-noise performance of two analog photonic links using different noise reduction techniques," in IEEE MTT-S Int. Microw. Symp. Dig., Honolulu, HI, Jun. 2007, pp. 51-54.

7. J.C. Campbell, et al., "High-power, high-linearity photodiodes for RF photonics," Conference on Optoelectronic and Microelectronic Materials \& Devices (COMMAD), pp. 215-216, 2012.

8. A. Karim and J. Devenport, "High dynamic range microwave photonic links for RF signal transport and RF-IF conversion," J. Lightwave Tech. 26 (15), p. 2718, 2008.

9. C. Middleton and R. DeSalvo, "Photonics-based wideband frequency conversion," Government Microcircuit Applications Critical Technology Conference (GOMAC), 2013.

10. A. Agarwal, et al. "Optically filtered microwave photonic links for RF signal processing applications" $J$. Lightwave Tech. 29 (16), p. 2394, 2011.

11. H. Lee, T. Chen, J. Li, O. Painter, and K.J. Vahala, "Ultra-low-loss optical delay line on a silicon chip," Nature Communications, 3 n. 867, 2012. 